\title{
Les noms de titre familial comme marqueurs de cohérence
}

\author{
Yumi Takagaki \\ Université préfectorale d'Osaka \\ takagaki@lc.osakafu-u.ac.jp
}

\section{Introduction}

Dans la première phrase de L'Étranger d'Albert Camus, reprise en (1), il y a une implication affective qui n'existe pas en (2). (Dans la suite de cet article, les caractères en gras apparaissant dans les exemples sont tous une mise en valeur voulue par nous, et non par l'auteur du texte original.)

(1) Aujourd'hui, maman est morte.

(2) Aujourd'hui, ma mère est morte.

Le mot maman est un indice énonciatif. Il donne l'impression de vivacité et d'intimité. Cet effet vient certainement de la nature même de ce nom, différent des noms « ordinaires ».

En effet, certains noms tels que Maman, Papa, Papi, Pépé, Mamie, Mémé ont des caractéristiques communes, différentes des autres noms désignant un membre familial. Il s'agit de termes d'adresse affectueux par lesquels les enfants, même devenus adultes, désignent un membre de leur famille. Nous les appelons noms de titre familial en les distinguant des noms de parenté ordinaires, tels que mère, père, grand-mère, grand-père, oncle, tante. Ces noms de titre familial sont, dans leur origine, des appellatifs. Mais ils s'emploient également sous forme de non-vocatif, tout comme les noms « ordinaires ». L'exemple suivant montre bien deux sortes de noms désignant une relation familiale.

(3) Je vis Moussa porté par deux militaires, il était pâle, très pâle, très affaibli. J'étais atterrée, consternée, mais j'ai demandé aux deux militaires où ils l'emmenaient ; ils daignèrent me répondre en précisant qu'ils le transportaient à l'hôpital. J'ai voulu les suivre, j'ai, bien entendu, reçu une réponse négative.

Dans le petit salon gisaient les corps de nos deux cousins morts. Les militaires nous firent descendre et le cauchemar continua. Au bas de l'escalier se trouvait le corps d'un des gardes de corps de papa, le sergent Badje. Plus loin le corps d'un oncle maternel, Moussa Kao, celui d'une tante de maman et d'autres... C'était vraiment une vision cauchemardesque et nous avions tous l'impression d'un cauchemar et que nous allions nous réveiller, mais hélas...

Il y a des noms de titre familial et des noms de parenté « ordinaires ». À la différence de ces derniers (cousins, oncle, tante), les noms de titre familial (papa, maman), dépourvus de déterminant, désignent automatiquement les parents de l'énonciateur, apparu dans le texte comme je. Qu'est-ce qui changerait dans ce texte si nous les remplacions par mon père et ma mère? Avec ces derniers, le récit prendrait un ton plus neutre. Par ailleurs, avec papa et maman le lecteur se sent plus «concerné ». Il y a un effet d'intimité, tout comme en (1).

À part cet effet, papa et maman en (3) sont respectivement interchangeables avec mon père et ma mère. Ce fait signifie-t-il que les noms de titre familial ne sont que des variantes stylistiques des noms de parenté précédés par un adjectif possessif de la première personne ? L'observation plus attentive des données montrera que ce n'est pas le cas: les noms de titre familial semblent avoir plusieurs caractéristiques différentes des noms « ordinaires » du point de vue textuel. 


\section{2 Études antérieures}

Les caractéristiques des termes d'adresse en français, y compris les noms de titre familial, ont été abordées en amont par plusieurs linguistes, dont notamment Perret (1968, 1970), Jaubert (1990, 2005), Lagorgette (1994, 2003, 2006) et Détrie (2006). Dans ces travaux, c'est surtout l'emploi vocatif (l'appellatif) qui a été étudié ; l'emploi non-vocatif dans le texte n'était pas une préoccupation majeure. Or l'emploi non-vocatif des noms de titre familial, illustré en (1) et (3), est non seulement fréquent, mais encore, à notre avis, doté de particularités linguistiques qui méritent d'être davantage examinées. Nous nous intéressons, dans cet article, à l'emploi non-vocatif des noms de titre familial, en particulier à leur fonction textuelle.

Nous pouvons pourtant rapprocher quelques caractéristiques des noms de titre familial et les caractéristiques générales des termes d'adresse. D'après Perret (1970), un terme d'adresse (qu'il appelle appellatif) peut être analysé sous les trois points de vue suivants :

- Il a un caractère déictique: il permet l'identification d'un référent (avec l'aide de toutes les indications que peut apporter la situation).

- Il a un caractère prédicatif : le sens de l'appellatif choisi, s'il en a un, et même si celui-ci est pauvre, permet d'effectuer une certaine prédication explicite.

- Il manifeste les relations sociales : par cela il permet d'effectuer une deuxième prédication, sousentendue, qui est celle de la relation sociale à la personne désignée.

Tout en adoptant et approfondissant ces trois points de vue, nous étudierons, dans ce qui suit, trois caractéristiques fondamentales des noms de titre familial : le caractère déictique, la référence dans les contextes de citation et le caractère performatif.

\section{Embrayeurs}

Comme l'observe Perret (1970), les noms de titre familial ont un caractère déictique : lorsqu'ils sont employés sans déterminant, ils renvoient à des membres de famille de l'énonciateur. Le mot maman, qui signifie nécessairement « ma mère », se comporte comme (incorporant) un déictique. Autrement dit, c'est un embrayeur. Les embrayeurs sont une classe de mots dont le sens varie avec la situation; ils ne peuvent être interprétés que s'ils sont rapportés à l'acte d'énonciation qui a produit l'énoncé où ils figurent.

L'exemple suivant montre deux sortes de maman.

(4) Tous les ans, c'est-à-dire le dernier et l'autre, parce qu'avant c'est trop vieux et je ne me rappelle pas, Papa et Maman se disputent beaucoup pour savoir où aller en vacances, et Maman se met à pleurer et elle dit qu'elle va aller chez sa maman, et moi, je pleure aussi parce que j'aime bien Mémé, mais chez elle il n'y a pas de plage, et à la fin on va où veut Maman et ce n'est pas chez Mémé. (Jean-Jacques Sempé et René Goscinny, Les Vacances du petit Nicolas, Denoël)

Le terme maman dans sa maman à la quatrième ligne est un nom de parenté « ordinaire »; c'est un synonyme de mère. Par ailleurs, les trois autres occurrences de Maman (sans déterminant, avec une majuscule) désignent systématiquement la mère de l'énonciateur. Autrement dit, ces trois Maman se comportent comme embrayeurs. La caractéristique identique s'observe aussi dans d'autres noms de titre familial.

Les noms de titre familial ne sont pourtant pas des embrayeurs « purs » au même titre que les embrayeurs comme je, ici, maintenant. En effet, en premier lieu, s'ils sont employés avec un déterminant, ils se comportent comme les noms « ordinaires ». Ils ont un statut intermédiaire entre les noms ordinaires et les embrayeurs «purs». En deuxième lieu, et ce qui importe le plus, c'est qu'à la différence de ces embrayeurs typiques, leur relation avec la situation d'énonciation est toujours indirecte. Ils se comportent comme incorporant un déictique, mais cette opération référentielle est indirecte. L'énonciateur manifeste certes sa présence dans l'énoncé. Mais il n'apparaît que par l'intermédiaire d'un membre de sa famille. Il 
y est inclus. Sa présence est ainsi toujours implicite. Cette introduction indirecte de l'énonciateur est, à notre avis, justement une fonction textuelle des noms de titre familial.

\section{Référence dans les contextes de citation}

Dire Papa ou Maman, ce n'est pas seulement interpeller quelqu'un, c'est lui dire comment on le considère. En ce sens, comme l'observe Perret (1970), une appellation inclut une prédication. Avec un nom de titre familial, l'énonciateur prédique explicitement sa relation familiale. Nous verrons que ce pouvoir de prédication est même plus fort dans son emploi non-vocatif.

Une des différences entre le vocatif et le non-vocatif se révèle dans des contextes de citation. L'emploi vocatif se caractérise par la non-intégration dans le discours indirect. Cette contrainte ne s'applique pas au non-vocatif.

(5) Sarah m'a dit : « Maman, tu es malade. »

(6) *Sarah m'a dit que, Maman, tu es malade.

(7) Sarah m'a dit que Maman était malade.

Or les noms de titre familial à l'emploi non-vocatif ont une caractéristique spéciale dans les contextes de citation. Ces contextes ont généralement (au moins) deux interprétations possibles avec un nom « ordinaire».

(8) Sarah m'a dit que ce vilain homme était malade.

La phrase en (8) est ambiguë entre l'interprétation de dicto et l'interprétation de re :

- interprétation de dicto: Le discours indirect est la transcription des paroles de Sarah, lesquelles seraient en discours direct : « Ce vilain homme est malade ».

- interprétation de re: C'est l'énonciateur, réalisé en (8) comme $m$ ', qui introduit les termes ce vilain homme. Il n'est pas nécessaire que Sarah les ait employés. Il est même possible qu'elle refuse pour elle-même toute validité aux termes : elle pense que l'homme en question est loin d'être vilain.

Cette ambiguïté peut être clarifiée par des conditions pragmatiques ou contextuelles. Mais la phrase (8) elle-même permet du moins deux interprétations.

Or, avec un nom de titre familial, seule l'interprétation de re se présente. Le terme Maman en (7), reproduit ci-dessous, ne désigne que la mère de l'énonciateur.

\section{(7)' Sarah m'a dit que Maman était malade.}

Pour rapporter la maladie de la mère de l'énonciateur (à moins que Sarah soit une sœur de l'énonciateur), Sarah ne peut pas avoir employé elle-même le terme Maman, qui désignerait automatiquement sa propre mère. L'énonciateur est forcé de reprendre le terme Maman à son compte. À la différence des noms « ordinaires », les noms de titre familial sont toujours liés à l'énonciateur, même dans les contextes de citations.

Pour mieux illustrer cette différence, imaginons la situation suivante : Hamlet, vexé du mariage hâtif de sa mère avec Claudius, vilain frère de son père, ne veut pas reconnaître que son oncle est devenu son (beau-) père ; il refuse de l'appeler papa, ou de le désigner par papa. Dans ce cas, il peut très bien énoncer les paroles en (9) :

(9) Rosencrantz m'a dit que mon père était en colère. Mais ce vilain homme n'est pas mon père. C'est l'époux de ma mère. C'est tout.

Le texte en (9) est cohérent lorsqu'on donne à la première phrase l'interprétation selon laquelle Rosencrantz a dit : «Votre père est en colère ». Dans la seconde phrase, Hamlet exprime son désaccord à cet emploi du mot père. 
À la différence de (9), les exemples (10) et (11) avec un nom de titre familial sont contradictoires (à moins que Rosencrantz soit un frère de Hamlet).

(10) 'Rosencrantz m'a dit que Papa était en colère. Mais ce vilain homme n'est pas mon père. C'est l'époux de ma mère. C'est tout.

(11) 'Rosencrantz m'a dit que Papa était en colère. Mais ce vilain homme n'est pas Papa. C'est l'époux de ma mère. C'est tout.

Avec un nom de titre familial, l'interprétation de dicto n'est pas possible: Rosencrantz ne peut pas employer le mot Papa pour désigner une personne qui n'est pas son parent. Dans la première phrase, en employant le terme Papa pour désigner ce vilain homme, l'énonciateur Hamlet reconnaît et consent au fait que celui-ci soit son père. Or il nie cette relation familiale tout de suite dans la seconde phrase. Cette inconsistance rend les enchaînements en (10) et en (11) bizarres. Ainsi les noms de titre familial ont-ils une spécificité que n'ont pas les noms « ordinaires ». Même lorsqu'ils sont cités dans les paroles d'autrui, ils sont automatiquement attribués à l'énonciateur.

Ce caractère transparent dans des contextes de citation est confirmé par l'emploi des guillemets. Si nous mettons entre guillemets papa de la première phrase de (10) et (11), l'acceptabilité s'améliore :

(12) Rosencrantz m'a dit que «papa » était en colère. Mais ce vilain homme n'est pas mon père. C'est l'époux de ma mère. C'est tout.

(13) Rosencrantz m'a dit que «papa » était en colère. Mais ce vilain homme n'est pas papa. C'est l'époux de ma mère. C'est tout.

C'est que les guillemets montrent graphiquement que les paroles qu'ils entourent ne doivent pas être attribuées à l'énonciateur; celui-ci refuse de reprendre les termes à son compte.

\section{Noms performatifs}

En appelant quelqu'un Maman, l'énonciateur reconnaît le fait que la personne en question est sa mère et qu'elle est digne d'être appelée ainsi. De ce fait, tout en refusant de construire une véritable relation familiale, la belle-mère de Blanche-Neige peut très bien dire à sa belle-fille : « Ne m'appelle pas Maman !» En effet, employer un nom de titre familial établit une relation. En ce sens, nous pouvons dire qu'il y a « une deuxième prédication sous-entendue » (Perret (1970), cité dans la section 2). Or, dans l'emploi non-vocatif, cette «deuxième prédication » des noms de titre familial rêvet, à notre avis, une autre signification que Perret n'a pas relevée.

Comme nous l'avons vu dans la section précédente, en employant un nom de titre familial l'énonciateur est obligé à consentir à la validité de ce terme. De ce fait, l'emploi d'un nom de titre familial constitue, par lui seul, un acte de récognition, reconnaissance, voire consentement. Or cette relation spécifique avec un acte de langage n'est pas sans exemples dans les langues : c'est elle que l'on décrit généralement à l'aide de la notion de performatif. Austin (1975) considère qu'il existe trois types d'actes de langage : l'acte locutoire, qui sous-tend toute énonciation par le simple fait que dire est en soi un acte ; l'acte illocutoire, qui accomplit une action par sa simple énonciation; et enfin l'acte perlocutoire, qui produit un effet sur l'allocutaire en proférant un énoncé. À notre avis, il existe dans les noms de titre familial des performatifs de reconnaissance. Nous pouvons rapprocher les noms de titre familial des verbes dits performatifs, tels promettre, s'engager, etc. ; comme la première personne du présent « je promets » par rapport à l'acte de promettre, les noms de titre familial fonctionnent par rapport à l'acte de récognition. Milner (1978) a fait une observation semblable en ce qui concerne les Noms de Qualité (idiot, imbécile, salaud, etc.). Il semble donc exister des noms performatifs ayant une force illocutoire, dont les noms de titre familial et les Noms de Qualité. Parallèlement aux phrases performatives, les noms performatifs se distinguent des noms constatifs par la caractéristique suivante : ils sont des actes ou, du moins, leur énonciation équivaut à l'accomplissement d'un acte.

Le caractère performatif des noms de titre familial est étroitement lié avec les deux caractères examinés dans les sections précédentes. Premièrement, il s'agit de son statut d'embrayeur. En principe, les verbes 
performatifs n'ont une valeur performative que lorsqu'ils sont employés avec un sujet de la première personne : «je promets» est un acte de promesse alors que «tu promets, il promet» ne le sont pas. La première personne a un statut privilégié dans les performatifs. Or le pronom de la première personne est un embrayeur par excellence. Deuxièmement, c'est un caractère prédicatif. Nous avons vu que le pouvoir de prédication d'un nom de titre familial est si fort qu'on peut prédiquer même dans le discours indirect. Cette force est certainement due à son caractère performatif. Comme l'observe Lakoff (1984), lorsqu'il y a une prédication dans une subordonnée celle-ci a une valeur illocutoire. Il nous semble qu'il en va de même pour les noms de titre familial.

La mise en relation entre les noms de titre familial et les énoncés illocutoires se retrouve déjà dans Lagorgette (2003). Celle-ci considère, d'après l'examen de textes en ancien et moyen français, que les termes d'adresse - y compris les noms de titre familial - sont des marqueurs d'acte de langage à deux niveaux locutoire/illocutoire. Lagorgette (2006) constate ensuite dans les termes d'adresse la capacité à modifier la valeur performative d'un énoncé. Détrie (2006) reprend la position de Lagorgette pour reconnaître dans les termes d'adresse le rôle de marqueur d'un acte de langage, et elle essaie de plus d'interpréter sa fonction dans l'organisation textuelle générale. Jaubert (2006) considère les termes d'adresse comme ayant la «valeur illocutoire indirecte : l'inflation des références culturelles fonctionne en soi comme un signe de reconnaissance ». Mais, même dans ces études, ce thème n'a pas été beaucoup approfondi. Par rapport à la relation entre les termes d'adresse et l'acte perlocutoire, soigneusement traitée par Perret (1968) et Lagorgette (1994), la relation avec l'acte illocutoire n'a pas suffisamment attiré l'attention de la plupart des linguistes.

\section{Empathie}

Les trois points de vue de Perret (1970) cités dans la section 2 peuvent être ainsi appliqués à notre analyse des noms de titre familial. En effet, les noms de titre familial sont des termes d'adresse. Ils ont pourtant une caractéristique particulière, étrangère à d'autres termes d'adresse. C'est leur éventuelle ambiguité référentielle dans le texte.

Nous avons constaté que, dépourvu de déterminant, le terme maman ne désigne que la mère de l'énonciateur. Or l'exemple suivant constitue un contre-exemple à cette observation.

(14) L'histoire de Sarah qui fouille dans le placard privé de Maman

Un matin, quand Maman fut partie,

Sarah se retrouva seule assise sur le tapis.

Elle eut une envie :

Celle de jouer avec le vernis.

Sarah entra dans la chambre des parents

Où se trouvait la coiffeuse de Maman. [... $]^{2}$

Cet exemple a deux interprétations possibles. Dans la première interprétation, le terme Maman fonctionne comme embrayeur : il désigne la mère de l'énonciateur du récit; c'est un équivalent de ma mère. On pourrait imaginer, par exemple, qu'un personnage appelé Sarah est une domestique profitant de l'absence de la maîtresse de maison et que l'enfant de celle-ci décrive l'acte clandestin de Sarah.

Dans cette interprétation, le terme Maman implique l'énonciateur. Et c'est seulement avec ce mot que le lecteur peut et doit sentir sa présence. Cette introduction indirecte de la première personne est, à notre avis, justement une fonction textuelle des noms de titre familial. Avec ceux-ci, il y a un mouvement de va-et-vient entre le texte et la situation d'énonciation, où se trouve l'énonciateur. Chaque occurrence des noms de titre familial relie le texte à la situation d'énonciation. En évoquant l'énonciateur qui prédique sa relation familiale et accomplit un acte de récognition, les noms de titre familial donnent une cohérence au texte. En effet, ils se comportent comme des ancres servant à fixer le texte par rapport au monde hors textuel.

Par ailleurs, le texte (14) a une autre interprétation, probablement plus saillante, qui pose toutefois un problème pour notre analyse. Le terme Maman désigne la mère de Sarah; c'est un équivalent de sa mère. 
Or Sarah n'est pas l'énonciatrice, mais un personnage du récit. Le terme Maman fonctionne comme un nom « ordinaire», tel que $(s a)$ mère. Cette seconde interprétation ne peut pas être expliquée par notre description ci-dessus des noms de titre familial. Pourquoi une telle référence est-elle possible en (14) ?

L'explication la plus plausible est de recourir à la notion de l'empathie, développée par Kuno (1987). D'après ce dernier, l'empathie est une identification de l'énonciateur à autrui, participant à l'événement ou à l'état qui se trouve décrit dans la phrase. C'est un phénomène largement observé dans diverses langues. Dans le cas de (14), l'énonciateur du récit s'identifie à Sarah, un personnage de son récit, pour (faire semblant de) ressentir ce qu'elle ressent et (faire semblant de) voir ce qu'elle voit. Il décrit ce que pense Sarah comme s'il était lui-même Sarah, tout en adoptant son point de vue. De ce fait, si Sarah appelle sa mère Maman, l'énonciateur aussi peut l'appeler Maman.

Si la notion d'empathie explique la référence non réflexive en (14), il faut quand même préciser les conditions pour savoir quand l'empathie a lieu. Car, à la différence de l'exemple en (14), elle n'est pas possible en (3), (4) et (7). Il faut expliquer pourquoi, par exemple en (7), reproduit ci-dessous, l'empathie permettant à l'énonciateur de s'identifier à Sarah n'est pas possible pour que Maman puisse désigner la mère de Sarah?

(7)" Sarah m'a dit que Maman était malade.

Afin d'éviter une application arbitraire de l'empathie, Kuno (1987) propose plusieurs hiérarchies d'empathie. Pour expliquer nos exemples, celle de l'acte de langage est pertinente :

Hiérarchie d'empathie de l'acte de langage

L'énonciateur ne peut pas avoir d'empathie pour quelqu'un d'autre davantage que pour lui-même.

$\mathrm{E}($ énonciateur $)>\mathrm{E}($ autres $)$

L'application de cette hiérarchie explique l'interprétation en (7). Dans cet exemple, l'énonciateur est lexicalement réalisé comme $m$ ', de sorte que la Hiérarchie d'empathie de l'acte de langage bloque l'empathie de l'énonciateur pour Sarah. Maman ne peut pas désigner la mère de Sarah.

Il en va de même pour (3), reproduit ci-dessous.

(3)' Je vis Moussa porté par deux militaires, il était pâle, très pâle, très affaibli. J'étais atterrée, consternée, mais j'ai demandé aux deux militaires où ils l'emmenaient ; ils daignèrent me répondre en précisant qu'ils le transportaient à l'hôpital. J'ai voulu les suivre, j'ai, bien entendu, reçu une réponse négative.

Dans le petit salon gisaient les corps de nos deux cousins morts. Les militaires nous firent descendre et le cauchemar continua. Au bas de l'escalier se trouvait le corps d'un des gardes de corps de papa, le sergent Badje. Plus loin le corps d'un oncle maternel, Moussa Kao, celui d'une tante de maman et d'autres... C'était vraiment une vision cauchemardesque et nous avions tous l'impression d'un cauchemar et que nous allions nous réveiller, mais hélas...

Dans ce texte, l'énonciateur, actualisé sous forme de je et $m e$, ne peut pas avoir d'empathie pour quelqu'un d'autre davantage que pour lui-même. Il ne peut donc pas s'identifier à d'autres personnages du récit. En effet, il raconte dans ce passage ce qu'il a vu de son point de vue; les personnages secondaires comme Moussa et les militaires ne sont pas le centre du point de vue. L'énonciateur ne peut pas prendre leurs positions pour appeler leurs parents papa et maman.

Il en va de même pour (4), reproduit ci-dessous. La présence de l'énonciateur, actualisé dans le même texte sous forme de je et $m e$, bloque l'empathie.

(4)' Tous les ans, c'est-à-dire le dernier et l'autre, parce qu'avant c'est trop vieux et je ne me rappelle pas, Papa et Maman se disputent beaucoup pour savoir où aller en vacances, et Maman se met à pleurer et elle dit qu'elle va aller chez sa maman, et moi, je pleure aussi parce que j'aime bien Mémé, mais chez elle il n'y a pas de plage, et à la fin on va où veut Maman et ce n'est pas chez Mémé. 
En revanche, en (14) reproduit ci-dessous, l'énonciateur n'apparaît pas dans le texte. Dans ce cas, la Hiérarchie d'empathie de l'acte de langage ne bloque pas l'empathie de l'énonciateur pour Sarah. S'il y a de l'empathie, Maman est la mère de Sarah. S'il n'y en a pas, Maman est la mère de l'énonciateur.

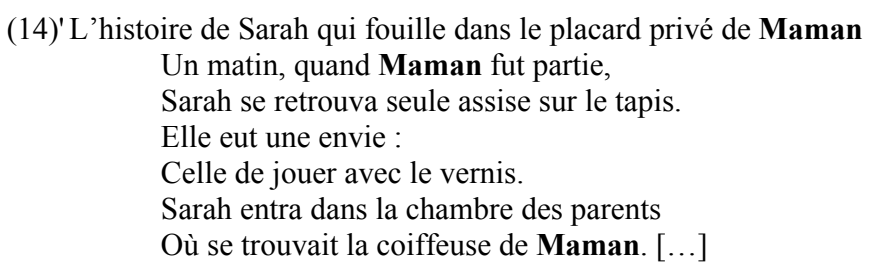

L'insertion de l'énonciateur je dans le texte change l'interprétation. L'exemple suivant est une réécriture de (14), avec des phrases contenant je.

(15) Je vous raconte l'histoire de Sarah qui fouille dans le placard privé de Maman. Un matin, quand Maman est partie, j'ai vu Sarah se retrouver seule dans la chambre de Maman. Je savais qu'elle avait une envie : celle de jouer avec le vernis de Maman.

En (15), il me semble plus difficile d'interpréter Maman comme la mère de Sarah (à moins que Sarah soit une sœur de l'énonciateur) : Maman désigne la mère de l'énonciateur réalisé dans le texte sous forme de je, qui est, par exemple, un enfant de la maîtresse de maison. (Et Sarah est une fille d'un employé de la maison.) Cette différence entre (14) et (15) suggère que la Hiérarchie d'empathie de l'acte de langage est pertinente.

En (1), reproduit ci-dessous, l'énonciateur est aussi implicite qu'en (14).

(1)' Aujourd'hui, maman est morte.

La Hiérarchie d'empathie de l'acte de langage ne s'applique pas. Pourtant, il n'y a pas l'ambiguïté, observée en (14). Car, à la différence de (14) contenant Sarah, cette phrase sans contexte n'a pas de candidat possible pour l'enfant de Maman. L'attribution à l'énonciateur est donc faite par le raisonnement par défaut. Maman ne peut désigner que la mère de l'énonciateur.

\section{Fonction textuelle}

L'explication recourant à l'empathie prédit, pourtant incorrectement, que l'exemple suivant est ambigu.

(16) Sarah a dit que Maman était malade.

De même que (1), l'énonciateur de la phrase en (16) n'apparaît pas dans le texte. La Hiérarchie d'empathie de l'acte de langage ne s'y applique pas. Il y a deux candidats possibles pour l'enfant de Maman: Sarah et l'énonciateur. D'après l'explication recourant à l'empathie, s'il y avait de l'empathie Maman serait la mère de Sarah et, s'il n'y en avait pas, Maman serait la mère de l'énonciateur. Or l'exemple (16) n'est pas ambigu. Le terme Maman ne peut désigner que la mère de l'énonciateur; l'empathie ne peut pas avoir lieu.

La phrase en (7), reproduite ci-dessous, est identique à celle en (16) sauf la présence de l'énonciateur réalisé comme $m$ '.

(7)" Sarah m'a dit que Maman était malade.

De même qu'en (16), Maman en (7) ne peut désigner que la mère de l'énonciateur ; l'empathie ne peut pas avoir lieu. Or l'explication recourant à l'empathie fait une prédiction erronée selon laquelle il y a une différence entre (7) et (16). Bref, l'empathie et la Hiérarchie d'empathie ne sont pas suffisantes pour expliquer toutes les interprétations possibles des noms de titre familial.

Or la phrase en (16) peut avoir l'interprétation que Maman est la mère de Sarah, s'il y a des contextes appropriés. Voici un texte semblable à (14). 

retrouvée seule et s'est mise à pleurer. Elle avait une envie : celle d'être toujours avec Maman. Son frère l'a retrouvée dans la chambre de Maman et lui a demandé pourquoi elle pleurait. Sarah a dit que Maman était malade.

À la différence de la phrase (16), qui est dépourvue de tout contexte, Maman dans la dernière phrase de (17) peut bien désigner la mère de Sarah. Contrairement à notre observation sur (7) de la section 4, s'il y a des contextes appropriés un nom de titre familial peut avoir une interprétation de dicto dans les contextes de citation. C'est grâce à l'empathie, qui enlève un nom de titre familial à ses caractéristiques fondamentales.

Les exemples suivants constituent aussi des cas problématiques pour l'explication recourant à l'empathie. L'exemple (18) est un texte posté sur un blog par une mère qui cherche des conseils auprès des participants du forum. Le texte (19) est un commentaire d'un membre du forum.

(18) Posté le 13-03-2007 à 10:27:29

Bonjour, j'ai un petit garçon de 4 ans. Il est en moyenne section et jusqu'à présent il aimait l'école. Mise à part sa toute première semaine en petite section, le temps de s'habituer. Mais depuis que sa maîtresse est revenue de congé maternité, il y a maintenant 3 semaines; il ne veut plus y aller. Alors que c'est la deuxième année qu'il a la même maîtresse.

La première chose qu'il me demande le matin, est « est-ce qu'il est revenu le maître ?» (celui-ci revient tous les vendredis car la maîtresse travaille à $80 \%$ ). Lorsque que je lui dis non, il me répond qu'il veut rester à la maison avec maman.

Les premiers jours, j'ai pris ça un peu pour un «caprice », mais hier midi, mon fils m'a dit qu'il avait pleuré le matin car il voulait maman. Et ce matin, au moment de laisser mes enfants dans la classe (ma fille de 3 ans et dans la même classe), mon fils s'est mis à pleurer en sortant de la classe. Je les accompagné de nouveau en tentant de le rassurer, lui disant que je revenais ce midi... Echec il a recommencé, je lui ai donné son doudou, qui reste habituellement dans le couloir, mais à peine le dos tourné que mon fils s'est mis à pleuré de plus belle. $[\ldots]^{3}$

(19) Posté le 13-03-2007 à 11:56:51

Et 30 secondes après il avait arrêté de pleurer parce qu'il sait que ce qui marche avec maman ne marche pas avec la maîtresse. ${ }^{4}$

L'interprétation de ces deux textes fait appel à une empathie. Les énonciateurs des textes en (18) et (19) s'identifient tous les deux à un petit garçon de 4 ans pour adopter son point de vue et pour appeler légitimement sa mère maman. En (19) l'énonciateur du texte n'étant pas actualisé, la Hiérarchie d'empathie de l'acte de langage ne s'y applique pas, de sorte que l'empathie peut avoir lieu. Maman désigne correctement la mère du garçon. En revanche, en (18) nos explications sur l'empathie ne peuvent pas rendre compte de l'interprétation. Car, à cause de la présence de l'énonciatrice dans le texte, réalisée sous forme de je et me, la Hiérarchie d'empathie de l'acte de langage devrait bloquer l'empathie. L'énonciatrice de (18) ne pourrait pas avoir d'empathie pour quelqu'un d'autre davantage que pour ellemême. Or ce n'est pas le cas pour (18). L'interprétation du texte exige que l'empathie ait lieu, alors que la Hiérarchie d'empathie de l'acte de langage la bloque incorrectement. Qu'est-ce qui différencie (18) de nos autres exemples de l'empathie?

La particularité de (18) réside dans le fait que le référent du nom de titre familial est le même que l'énonciatrice du texte. Il semble que dans ce cas, la Hiérarchie d'empathie de l'acte de langage ne s'applique pas. Nous vérifierons cette hypothèse avec l'exemple suivant, qui est une réécriture de (15), avec une phrase précisant que Sarah est une fille de l'énonciatrice, réalisée comme je.

(20) Je vous raconte l'histoire de Sarah qui fouille dans le placard privé de Maman. Un matin, j'ai vu ma fille Sarah se retrouver seule dans la chambre 
de Maman. Je savais qu'elle avait une envie : celle de jouer avec le vernis de Maman.

À la différence de (15), Maman en (20) peut désigner l'énonciatrice réalisée comme je, qui est la mère de Sarah. Cette différence entre (15) et (20) suggère que la Hiérarchie d'empathie de l'acte de langage ne s'applique pas lorsque le nom de titre familial désigne l'énonciateur du texte.

Revenons à l'exemple (18). Étant donné la situation d'énonciation en question, nous savons que maman en (18) désigne l'énonciatrice elle-même. Mais, dépourvu du contexte et avec de petites modifications du texte, maman en (18) aurait, avec une empathie, un autre référent. Imaginons que la première phrase soit « Bonjour, je suis nourrice d'un petit garçon de 4 ans. » ou « Bonjour, je m'occupe d'un petit garçon de 4 ans. » et remplaçons dans le troisième paragraphe les deux occurrences de mon fils par il. Dans ce cas, le terme maman désignerait seulement la mère du garçon, laquelle n'est pas l'énonciatrice. Voilà une seconde interprétation avec une empathie.

Il peut y avoir une troisième interprétation, sans que l'empathie ait lieu. Il suffit d'imaginer une situation où l'énonciateur est un fils d'une nourrice s'occupant d'un enfant gâté de sa patronne. Remplaçons la première phrase en (18) par «Bonjour. J'ai ma mère qui est nourrice. Elle et moi devons nous occuper ensemble d'un petit garçon de 4 ans. » et substituons $i l$ aux deux occurrences de mon fils dans le troisième paragraphe. Dans ce cas, maman en (18) pourrait désigner la mère de l'énonciateur.

Nous avons vu avec l'exemple (18) trois interprétations possibles d'un nom de titre.

- L'empathie n'a pas lieu; le référent du nom de titre familial est un membre de famille de l'énonciateur. (Cas canonique d'un nom de titre familial.)

- L'empathie a lieu; le référent du nom de titre familial est égal à un membre de famille d'un personnage dans le texte, mais pas à l'énonciateur. (Cas canonique de l'empathie.)

- L'empathie a lieu ; le référent du nom de titre familial est égal à l'énonciateur.

Le fait qu'il existe trois interprétations possibles suggère un caractère complexe des noms de titre familial. Ils sont des porteurs de l'ambiguïté, à cause de l'empathie.

\section{Conclusion et remarque}

Voici les caractéristiques des noms de titre familial que nous avons observées dans cet article.

- Ils ont un effet d'intimité.

- Ils ont un statut intermédiaire entre les embrayeurs « purs » et les noms « ordinaires ».

- Ils ont normalement une interprétation de re dans des contextes de citation.

- Ils sont des noms performatifs, en ce sens que l'utilisation de ces termes à elle seule constitue un acte de récognition.

- Ils sont d'éventuels porteurs d'une ambiguïté référentielle à cause de l'empathie.

Avant de terminer, il faut ajouter une remarque importante. Dans la discussion de la section 5, nous avons traité la force illocutoire comme si on pouvait distinguer clairement les noms performatifs et les noms constatifs. Or, comme le remarquent bien Récanati (1979) et Ruwet (1982), il est impossible d'établir une frontière nette entre ces deux notions. Il faut imaginer plutôt un continuum. En effet, quelques caractéristiques des noms de titre familial décrites dans cet article sont aussi partagées par d'autres termes d'adresse, tels que les noms de titre professionnel (chauffeur, professeur, etc.), les termes d'injure (imbécile, idiot, etc.), les termes relationnels (ami, camarade, etc.). Les différents degrés de performativité entre ces noms seront l'objet d'une autre étude constituant une étude systématique de tous les termes d'adresse. 
* La présente recherche bénéficie de l'Aide pour la recherche scientifique (C) de KAKENHI (21520446), accordée par la Société japonaise pour la promotion des sciences (JSPS).

\section{Références bibliographiques}

Austin, J. L. (1975). How to do Things with Words. London : Oxford University Press.

Benveniste, É. (1966). Problèmes de linguistique générale 1. Paris : Gallimard.

Braun, F. (1988). Terms of Address: Problems of Patterns and Usage in Various Languages and Cultures. Berlin : Mouton de Gruyter.

Détrie, C. (2006). De la non-personne à la personne : l'apostrophe nominale. Paris : CNRS Éditions.

Ducrot, O. (1984). Le Dire et le Dit. Paris : Minuit.

Jakobson, R. (1957[1963]). Les embrayeurs, les catégories verbales et le verbe russe. In Jacobson, R., Essais de linguistique générale. Paris : Minuit, 176-196.

Jaubert, A. (1988). L'énonciation réflexive en première ligne, dessin de l'acte de parole. Langue française, 79 : 64-81.

Jaubert, A. (1990). La lecture pragmatique. Paris : Hachette.

Jaubert, A. (2005). Négociation de la mise en places et stratégies de l'idéalisation. Semen, 20, $<$ http://semen.revues.org/document2032.html. Consulté le 13 mars 2010>.

Kerbrat-Orecchioni, C. (1999). L'énonciation. Paris : Armand Colin.

Kerbrat-Orecchioni, C. (2008). Les actes de langage dans le discours : théories et fonctionnement. Paris : Armand Colin.

Kuno, S. (1987). Functional Syntax: Anaphora, Discouse and Empathy. Chicago : The University of Chicago Press.

Kuroda, S.-Y. (1976). Reflection on the Foundations of Narrative Theory from a Linguistic Point of View. In van. Dijk, T. A. (éd.), Pragmatics of Language and Literature. Amsterdam : North-Holland Publishing Company, 107-140.

Kuroda, S.-Y. (1979). Réflexions sur les fondements de la théorie de la narration. In Kristeva, J., Milner, J.-C. et Ruwet, N. (éds.), Langue, discours, société : pour Émile Benveniste. Paris : Seuil, 260-293

Kuroda, S.-Y. (1979). Où l'épistémologie, la grammaire et le style se rencontrent : examen d'un exemple japonais. In S.-Y. Kuroda, Aux quatre coins de la linguistique. Paris : Seuil, 235-259.

Kuroda, S.-Y. (1979). Grammaire et récit. In Kuroda, S.-Y., Aux quatre coins de la linguistique. Paris : Seuil, 261271.

Lagorgette, D. (1994). Termes d'adresse, acte perlocutoire et insultes : la violence verbale dans quelques textes des $14^{\mathrm{e}}, 15^{\mathrm{e}}$ et $16^{\mathrm{e}}$ siècles. Senefiance, $36,317-332$.

Lagorgette, D. (2003). Termes d'adresse, insulte et notion de détachement en diachronie : quels critères d'analyse pour la fonction d'adresse ? Cahiers de praxématique, 40, 43-69.

Lagorgette, D. (2006). Du vocatif à l'apostrophe : problèmes terminologiques et théoriques. Termes d'adresse et détachement en diachronie du français. L'information grammaticale, 109, 38-44.

Lakoff, G. (1984). Performative subordinate clauses. BLS, 10, 472-80.

Milner, J.-C. (1978). De la syntaxe à l'interprétation. Paris : Seuil.

Perret, D. (1968). Termes d'adresse et injures. Cahiers de lexicologie, 12, 3-14.

Perret, D. (1970). Les appellatifs : analyse lexicale des actes de parole. Langages, 17, 112-118.

Récanati, F. (1979). La transparence et l'énonciation : pour introduire à la pragmatique. Paris : Seuil.

Récanati, F. (1981). Les énoncés performatifs. Paris : Minuit. 
Ross, J. R. (1970). On Declarative Sentences. Readings. In Jacobs, R. A. et Rosenbaum, P. S. (éds) Englich Transformational Grammar. Waltham Mass. : Ginn, 222-272.

Ruwet, N. (1982). Grammaire des insultes. In Ruwet, N., Grammaire des insultes et autres études. Paris : Seuil, 239314.

Searle, J. R. (1969). Speech Acts: an Essay in the Philosopy of Language. London : Cambridge University Press.

Takagaki, Y. (2008). Les plans d'organisation textuelle en français et en japonais : de la rhétorique contrastive à la linguistique textuelle, thèse de doctorat en sciences du langage, Rouen, université de Rouen, $<\mathrm{http}: / /$ tel.archivesouvertes.fr/tel-00349416/fr/>.

Takagaki, Y. (à paraître en 2011). De la rhétorique contrastive à la linguistique textuelle : l'organisation textuelle en français et en japonais, chapitre 9, Osaka et Rouen: Osaka Municipal Universities Press \& Publications des universités de Rouen et du Havre.

${ }^{1}<$ http://www.koinai.net/sur-le-vif/niger-1974-ce-qui-n-a-pas-ete-dit/article/moussa-est-blesse>, Consulté le 3 avril 2010.

${ }^{2}<$ http://pharouest.ac-rennes.fr/e221560B/crassetignasse/histoiredeSarah.htm>, Consulté le 3 avril 2010.

${ }^{3}<$ http://forum.magicmaman.com/magic37ans/ecole-maternelle-cp/plus-aller-ecole-sujet-3661892-1.htm\#t3696254>, Consulté le 3 avril 2010.

${ }^{4}<\mathrm{http}$ //forum.magicmaman.com/magic37ans/ecole-maternelle-cp/plus-aller-ecole-sujet-3661892-1.htm\#t3696254>, Consulté le 3 avril 2010. 